\title{
Total Positivity of the Cubic Trigonometric Bézier Basis
}

\author{
Xuli Han and Yuanpeng Zhu \\ School of Mathematics and Statistics, Central South University, Changsha 410083, China \\ Correspondence should be addressed to Yuanpeng Zhu; zhuyuanpeng@csu.edu.cn \\ Received 12 September 2013; Revised 9 February 2014; Accepted 12 February 2014; Published 17 March 2014 \\ Academic Editor: Nicola Mastronardi
}

Copyright (C) 2014 X. Han and Y. Zhu. This is an open access article distributed under the Creative Commons Attribution License, which permits unrestricted use, distribution, and reproduction in any medium, provided the original work is properly cited.

Within the general framework of Quasi Extended Chebyshev space, we prove that the cubic trigonometric Bézier basis with two shape parameters $\lambda$ and $\mu$ given in Han et al. (2009) forms an optimal normalized totally positive basis for $\lambda, \mu \in(-2,1]$. Moreover, we show that for $\lambda=-2$ or $\mu=-2$ the basis is not suited for curve design from the blossom point of view. In order to compute the corresponding cubic trigonometric Bézier curves stably and efficiently, we also develop a new corner cutting algorithm.

\section{Introduction}

Trigonometric splines and polynomials have attracted widespread interest within computer aided geometric design (CAGD), particularly within curve design. In [1], the recurrence relation for the trigonometric B-splines of arbitrary order was established. Later, in [2], it was further shown that the trigonometric B-splines of odd order form a partition of a constant in the case of equidistant knots, and thus the associated trigonometric B-spline curve possesses the convex hull property. In [3], a family of trigonometric polynomials was introduced, which contains the trigonometric Lagrange and Bernstein polynomials. The study for the C-Bézier and C-B-spline curves can be found in [4-6]. In [7], the totally positive property of the C-B-spline was proved. In [8-11], some quadratic trigonometric polynomial splines with shape parameters were shown. In [12], a class of cubic trigonometric Bézier (T-Bézier, for short) basis with a shape parameter was proposed. In [13], the cubic T-Bézier basis was further extended to possess two shape parameters. Recently, based on the theory of envelop and topological mapping, shape analysis of the cubic T-Bézier curve with shape parameters was given in $[14,15]$. For the problems of shape preserving interpolation, the cubic and quadratic T-Bézier bases show great potential applications; see [16-19]. Blossom is a powerful tool for studying Bézier-like bases and B-spline-like bases. Quasi Extended Chebyshev (QEC) space is the largest class of spaces of sufficient regularity suited for curve design in the sense that they do possess blossom. See the recently developed theory concerning blossom and QEC-space given in [20-24].

Many shape preserving properties are obtained when the normalized bases are totally positive; see [25]. For instance, the length, number of inflections, and angular variation of the curve defined by normalized totally positive bases are bounded above by those of the control polygon. The optimal normalized totally positive basis (i.e., the normalized B-basis) is the unique normalized basis of a space with optimal shape preserving properties. It is well known that the Bernstein basis is the optimal normalized totally positive basis of the space of polynomials of degree less than or equal to $n$; see [26]. Corner cutting algorithm plays a vital role in CADG since it provides simple geometric constructions of curves; see [27]. A relevant example is the de Casteljau algorithm for the evaluation of a polynomial curve using the Bernstein basis. The purpose of this paper is to prove that the cubic trigonometric Bézier basis with two shape parameters $\lambda$ and $\mu$ given in [13] forms an optimal normalized totally positive basis for $\lambda, \mu \in(-2,1]$ and to show a new developed corner cutting algorithm for computing the corresponding cubic trigonometric Bézier curves.

The rest of this paper is organized as follows. Section 2 gives the proof of the optimal normalized totally positive property of the cubic trigonometric Bézier basis within the general framework of QEC-space. In Section 3, a new developed corner cutting algorithm for computing the corresponding cubic trigonometric Bézier curves is shown. Conclusions are given in Section 4. 


\section{Cubic Trigonometric Bézier Basis Functions}

2.1. Preliminaries. Here, we give the necessary background on Extended Completed Chebyshev (ECC) space and Quasi Extended Chebyshev (QEC) space. Few results are described briefly for a good understanding to this paper, and more details can be found in [20-24, 28].

Let $I$ denote a given closed bounded interval $[a, b]$, with $a<b$. We call function space $\left(u_{0}, \ldots, u_{n}\right)$ a $n+1$-dimension ECC-space in canonical form generated by positive weight functions $w_{j} \in C^{n-j}(I)$ provided that

$u_{0}(t)=w_{0}(t)$,

$u_{1}(t)=w_{0}(t) \int_{a}^{t} w_{1}\left(t_{1}\right) d t_{1}$,

$u_{2}(t)=w_{0}(t) \int_{a}^{t} w_{1}\left(t_{1}\right) \int_{a}^{t_{1}} w_{2}\left(t_{2}\right) d t_{2} d t_{1}$,

$u_{n}(t)=w_{0}(t) \int_{a}^{t} w_{1}\left(t_{1}\right) \int_{a}^{t_{1}} w_{2}\left(t_{2}\right) \cdots \int_{a}^{t_{n-1}} w_{n}\left(t_{n}\right) d t_{n} \cdots d t_{1}$.

It is well known that $(n+1)$-dimension function space $\left(u_{0}, \ldots, u_{n}\right) \subset C^{n}(I)$ is an ECC-space on $I$ if and only if for any $k, 0 \leq k \leq n$, any nonzero element of the subspace $\left(u_{0}, \ldots, u_{k}\right)$ has at most $k$ zeros (counting multiplicities). And $(n+1)$ dimension function space $\left(u_{0}, \ldots, u_{n}\right) \subset C^{n-1}(I)$ is a QECspace on $I$ if any nonzero element of the space vanishes at most $n$ times in $I$, counting multiplicities as far as possible for functions in $C^{n-1}(I)$, that is, up to $n$; see [21-24].

A basis $\left(u_{0}, \ldots, u_{n}\right)$ is said to be normalized on $[a, b]$ if $\sum_{i=0}^{n} u_{i}(t)=1$ for any $t \in[a, b]$. And a basis $\left(u_{0}, \ldots, u_{n}\right)$ is said to be totally positive on $[a, b]$ if, for any sequence of points $a \leq t_{0}<t_{1}<\cdots<t_{n} \leq b$, the collocation matrix $\left(u_{j}\left(t_{i}\right)\right)_{0 \leq i, j \leq n}$ is totally positive; that is, all its minors are nonnegative. For a given function space possessing totally positive basis, the optimal normalized totally positive basis (i.e., the normalized B-basis) is a normalized totally positive basis from which all other totally positive bases can be deduced by multiplying with a (regular) totally positive matrix. Such optimal normalized totally positive basis is unique and has optimal shape preserving properties (see [25]) in the sense that the curves defined by such basis best imitate the corresponding control polygons (e.g., the monotonicity and convexity of the control polygon are inherited by the curves defined by such basis).

2.2. Definition of the Cubic Trigonometric Bézier Basis. Here, we recall the definition of the cubic trigonometric Bézier basis with two shape parameters given in [13] as follows.

Definition 1. Let $\lambda, \mu \in[-2,1]$, for $t \in[0, \pi / 2]$; the following four functions are defined to be the cubic trigonometric Bézier (T-Bézier) basis functions, with two shape parameters $\lambda$ and $\mu$ :

$$
\begin{aligned}
& T_{0}(t)=(1-\sin t)^{2}(1-\lambda \sin t), \\
& T_{1}(t)=\sin t(1-\sin t)(2+\lambda-\lambda \sin t),
\end{aligned}
$$

$$
\begin{aligned}
& T_{2}(t)=\cos t(1-\cos t)(2+\mu-\mu \cos t), \\
& T_{3}(t)=(1-\cos t)^{2}(1-\mu \cos t) .
\end{aligned}
$$

Specially, for $\lambda=\mu$, this kind of T-Bézier basis with a shape parameter was also proposed in [12].

By rewriting the expressions of $T_{1}(t)$ and $T_{2}(t)$ as the following forms:

$$
\begin{aligned}
& T_{1}(t)=1-\sin ^{2} t-(1-\sin t)^{2}(1-\lambda \sin t), \\
& T_{2}(t)=1-\cos ^{2} t-(1-\cos t)^{2}(1-\mu \cos t),
\end{aligned}
$$

we can easily see that the cubic T-Bézier basis (2) is normalized and is actually a basis of the following trigonometric function space:

$$
\begin{gathered}
T_{\lambda, \mu}:=\operatorname{Span}\left\{1, \sin ^{2} t,(1-\sin t)^{2}(1-\lambda \sin t),\right. \\
\left.(1-\cos t)^{2}(1-\mu \cos t)\right\} .
\end{gathered}
$$

2.3. Optimal Totally Positive Property of the Basis. Firstly, we want to show that for any $\lambda, \mu \in(-2,1]$, the following space

$$
\begin{aligned}
& D T_{\lambda, \mu} \\
& :=\operatorname{Span}\{2 \sin t \cos t,-\cos t(1-\sin t)(2+\lambda-3 \lambda \sin t), \\
& \quad \sin t(1-\cos t)(2+\mu-3 \mu \cos t)\}
\end{aligned}
$$

is a 3-dimensional QEC-space on $[0, \pi / 2]$.

Theorem 2. For any real numbers $\lambda, \mu \in(-2,1]$, the space $D T_{\lambda, \mu}$ is a 3-dimensional QEC-space on $[0, \pi / 2]$.

Proof. For any $\xi_{i} \in \mathbb{R}, \lambda, \mu \in(-2,1]$, and $t \in[0, \pi / 2]$, we consider a linear combination:

$$
\begin{aligned}
& \xi_{0}(2 \sin t \cos t)+\xi_{1}[-\cos t(1-\sin t)(2+\lambda-3 \lambda \sin t)] \\
& +\xi_{2}[\sin t(1-\cos t)(2+\mu-3 \mu \cos t)]=0 .
\end{aligned}
$$

For $t=0$, from (6), we can immediately obtain $\xi_{1}=0$. Similarly, for $t=\pi / 2$, from (6), we have $\xi_{2}=0$. And finally, we have $\xi_{0}=0$. Thus the space $D T_{\lambda, \mu}$ is a 3 -dimensional space.

Now, we want to prove that the space $D T_{\lambda, \mu}$ is a 3dimensional ECC-space in $(0, \pi / 2)$. For any $t \in[a, b] \subset$ $(0, \pi / 2)$, let

$$
\begin{aligned}
u(t) & =\left[-\frac{(1-\sin t)(2+\lambda-3 \lambda \sin t)}{\sin t}\right]^{\prime} \\
& =\frac{\cos t}{\sin ^{2} t}\left(2+\lambda-3 \lambda \sin ^{2} t\right), \\
v(t) & =\left[\frac{(1-\cos t)(2+\lambda-3 \lambda \cos t)}{\cos t}\right]^{\prime} \\
& =\frac{\sin t}{\cos ^{2} t}\left(2+\mu-3 \mu \cos ^{2} t\right) .
\end{aligned}
$$


For any $\lambda, \mu \in(-2,1]$, it is obvious that $u(t)>0$ and $v(t)>0$. By directly computing, we have

$$
\begin{aligned}
& u^{\prime}(t)=-\frac{1}{\sin ^{3} t}\left[\left(2+\lambda-3 \lambda \sin ^{2} t\right) \sin ^{2} t+2(2+\lambda) \cos ^{2} t\right]<0, \\
& v^{\prime}(t)=\frac{1}{\cos ^{3} t}\left[\left(2+\mu-3 \mu \cos ^{2} t\right) \cos ^{2} t+2(2+\mu) \sin ^{2} t\right]>0 .
\end{aligned}
$$

Therefore, for the Wronskian of $u$ and $v$, we have

$$
W(u, v)(t)=u(t) v^{\prime}(t)-u^{\prime}(t) v(t)>0, \quad \forall t \in[a, b] .
$$

For $t \in[a, b]$, we define the following weight functions:

$$
\begin{aligned}
& w_{0}(t)=2 \sin t \cos t, \\
& w_{1}(t)=A u(t)+B v(t), \\
& w_{2}(t)=C \frac{W(u, v)(t)}{[A u(t)+B v(t)]^{2}},
\end{aligned}
$$

where $A, B$, and $C$ are three arbitrary positive real numbers. Obviously, these weight functions $w_{i}(t)(i=0,1,2)$ are bounded, positive, and $C^{\infty}$ on $[a, b]$. Consider the following ECC-space defined by the weight functions $w_{i}(t)(i=0,1,2)$ :

$$
\begin{aligned}
& u_{0}(t)=w_{0}(t) \\
& u_{1}(t)=w_{0}(t) \int_{a}^{t} w_{1}\left(t_{1}\right) d t_{1}, \\
& u_{2}(t)=w_{0}(t) \int_{a}^{t} w_{1}\left(t_{1}\right) \int_{a}^{t_{1}} w_{2}\left(t_{2}\right) d t_{2} d t_{1} .
\end{aligned}
$$

After some simple computations, we can see that these functions $u_{i}, i=0,1,2$ are in fact some linear combinations of the three functions $2 \sin t \cos t,-\cos t(1-\sin t)(2+\lambda-$ $3 \lambda \sin t)$, and $\sin t(1-\cos t)(2+\mu-3 \mu \cos t)$, which indicates that the space $D T_{\lambda, \mu}$ is a ECC-space on $[a, b]$. Since $[a, b]$ is arbitrary subinterval of $(0, \pi / 2)$, we can conclude that the space $D T_{\lambda, \mu}$ is an ECC-space in $(0, \pi / 2)$. Now, we want to further prove that the space $D T_{\lambda, \mu}$ is also a QEC-space on $[0, \pi / 2]$. For this purpose, we need to prove that any nonzero element of the space $D T_{\lambda, \mu}$ has at most 2 zeroes on $[0, \pi / 2]$ (counting multiplicities as far as possible up to 2). Consider any nonzero function:

$$
\begin{aligned}
F(t)= & C_{0}(2 \sin t \cos t) \\
& +C_{1}[-\cos t(1-\sin t)(2+\lambda-3 \lambda \sin t)] \\
& +C_{2}[\sin t(1-\cos t)(2+\mu-3 \mu \cos t)],
\end{aligned}
$$

where $t \in[0, \pi / 2]$. Since the space $D T_{\lambda, \mu}$ is an ECC-space in $(0, \pi / 2)$, the function $F(t)$ has at most two zeroes in $(0, \pi / 2)$. Let us consider a case that $F(t)$ vanishes at 0 ; then, we have $C_{1}=0$. For this case, if $C_{2}=0$, then $F(t)$ has a singular zero at 0 and a singular zero at $\pi / 2$. If $C_{0}=0$, it can be easily checked that 0 is a double zero of $F(t)$ (counting multiplicities as far as possible up to 2). If $C_{0} C_{2}>0, F(t)$ vanishes exactly one time at 0 and it does not vanish on $(0, \pi / 2]$. If $C_{0} C_{2}<0, F(t)$ vanishes exactly one time at 0 and it does not vanish at $\pi / 2$. Moreover, consider the following function:

$$
\begin{array}{r}
G(t)=2 C_{0} \cos t+C_{2}[(1-\cos t)(2+\mu-3 \mu \cos t)], \\
t \in\left[0, \frac{\pi}{2}\right] .
\end{array}
$$

Direct computation gives that $G^{\prime}(t)=\sin t K(t)$, where $K(t)=$ $-2 C_{0}+C_{2}(2+4 \mu-6 \mu \cos t)$. Obviously, $K(t)$ is a monotonic function on $[0, \pi / 2]$, and thus $K(t)$ has at most one zero in $(0, \pi / 2)$, which implies that $G^{\prime}(t)$ also has at most one zero in $(0, \pi / 2)$. From these together with $G(0) G(\pi / 2)=$ $2(2+\mu) C_{0} C_{2}<0$, we can see that $G(t)$ has exactly one zero in $(0, \pi / 2)$, and thus we can immediately conclude that $F(t)=\sin t G(t)$ (notice that $C_{1}=0$ for the current case) has exactly one zero in $(0, \pi / 2)$ too. Similarly, for the case that $F(t)$ vanishes at $\pi / 2$, we can also deduce that the function $F(t)$ has at most 2 zeroes on $[0, \pi / 2]$ (counting multiplicities as far as possible up to 2). To summarize, the space $D T_{\lambda, \mu}$ is a QECspace on $[0, \pi / 2]$.

Remark 3. By Theorem 3.1 of [22], we can see that blossom exists in the trigonometric function space $T_{\lambda, \mu}$ is equivalent to that the space $D T_{\lambda, \mu}$ is a QEC-space. For any $\lambda, \mu \in(-2,1]$, since the space $D T_{\lambda, \mu}$ is a QEC-space on $[0, \pi / 2]$, blossom exists in $T_{\lambda, \mu}$. By Theorem 2.13 of [22], we can also see that the space $T_{\lambda, \mu}$ possesses a normalized Quasi Bernstein-like basis on $[0, \pi / 2]$ for $\lambda, \mu \in(-2,1]$.

It is worth mentioning that for the special case $\lambda=-2$ or $\mu=-2, F(t)$ may have three distinct zeros on $[0, \pi / 2]$. For example, for $\mu=-2, C_{0}=3, C_{1}=0$, and $C_{2}=-2, F(t)$ will vanish at $0, \pi / 3$ and $\pi / 2$, respectively, which implies that $D T_{\lambda, \mu}$ is not a QEC-space on $[0, \pi / 2]$ for $\lambda=-2$ or $\mu=-2$. Thus, from the blossom point of view, $T_{\lambda, \mu}$ is not suited for curve design for the case that $\lambda=-2$ or $\mu=-2$.

Theorem 4. For any $\lambda, \mu \in(-2,1]$, the cubic T-Bézier basis given in (2) is the optimal normalized totally positive basis of the space $T_{\lambda, \mu}$.

Proof. For any $\lambda, \mu \in(-2,1]$, from the expressions of the four cubic T-Bézier basis functions given in (2), we can easily check the following end-point properties of the basis.

(i) $T_{0}(0)=1$, and $T_{0}(t)$ vanishes 3 times at $\pi / 2$ (counting multiplicities as far as possible up to 3 ).

(ii) $T_{3}(\pi / 2)=1$, and $T_{3}(t)$ vanishes 3 times at 0 (counting multiplicities as far as possible up to 3 ).

(iii) For $i=1,2, T_{i}(t)$ vanishes exactly $i$ times at 0 and exactly $3-i$ times at $\pi / 2$.

In addition, $T_{i}(t)(i=0,1,2,3)$ is strictly positive in $(0, \pi / 2)$. Thus, by Definition 2.10 of [22], we can see that the cubic T-Bézier basis (2) is precisely the Quasi Bernstein-like basis of the space $T_{\lambda, \mu}$. And by Theorem 2.18 of [22], we can further conclude that the cubic T-Bézier basis (2) is exactly the optimal normalized totally positive basis of the space $T_{\lambda, \mu}$. 


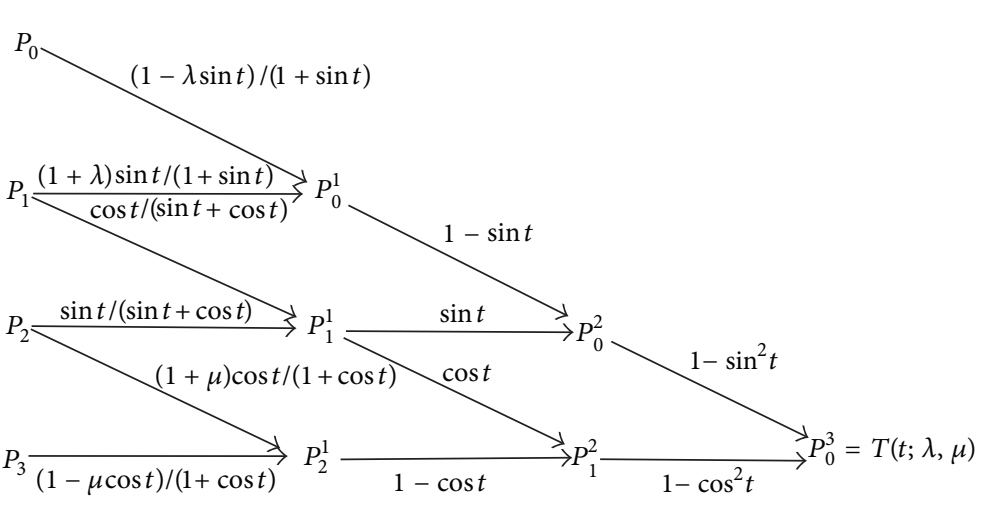

(a)

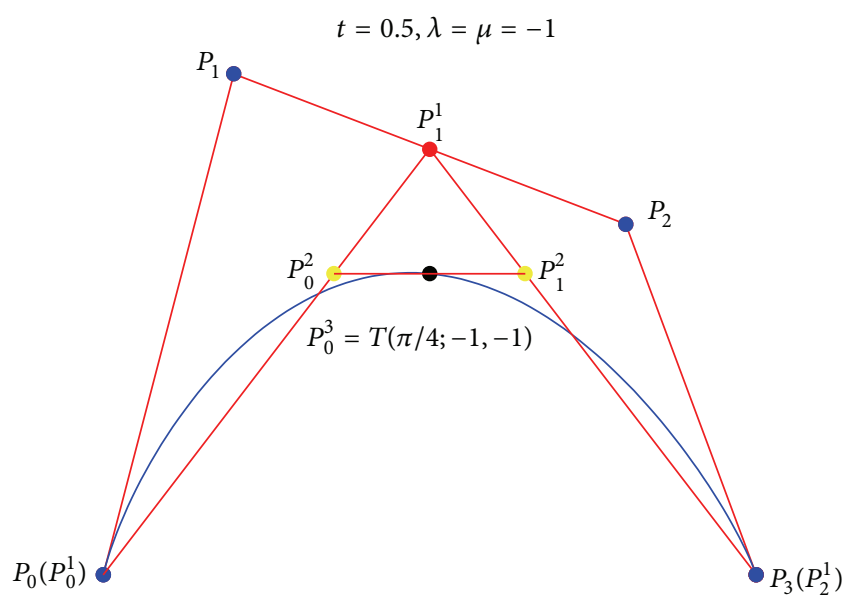

(c)

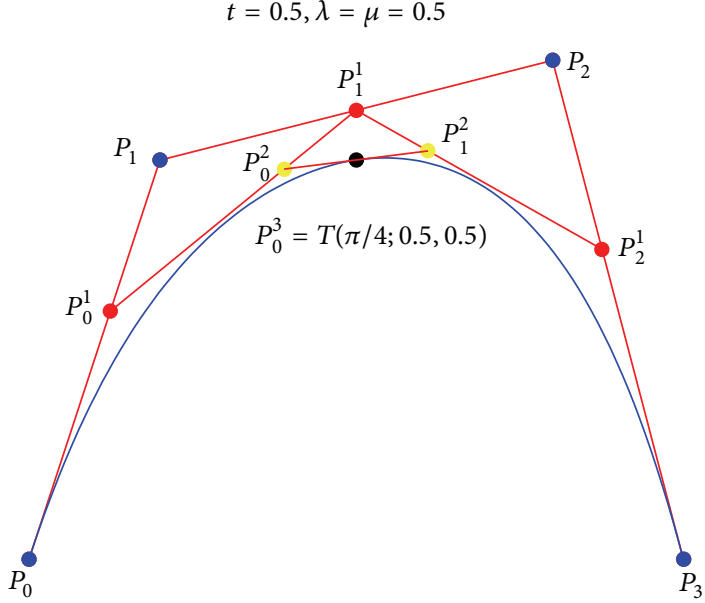

(b)

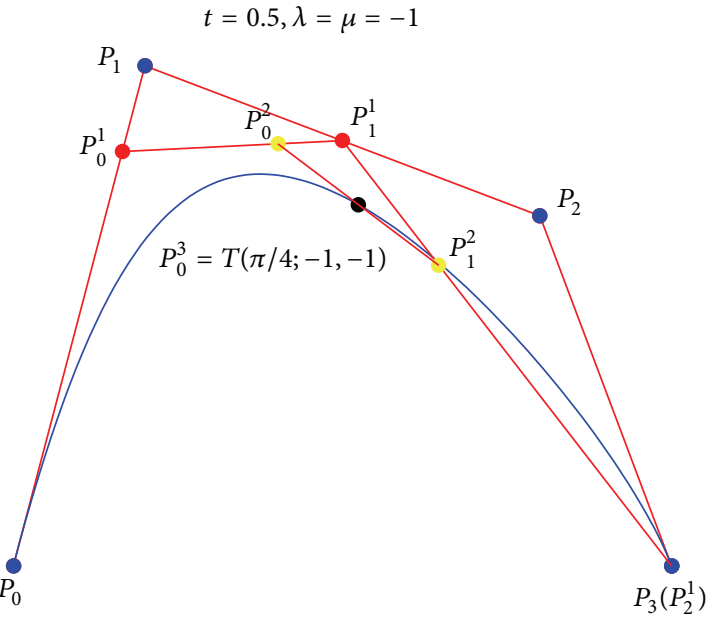

(d)

FIGURE 1: Corner cutting algorithm.

\section{Cubic Trigonometric Bézier Curve}

Definition 5. Given control points $P_{i}(i=0,1,2,3)$ in $\mathbb{R}^{2}$ or $\mathbb{R}^{3}$, then

$$
T(t ; \lambda, \mu)=\sum_{i=0}^{3} T_{i}(t) P_{i}, \quad t \in\left[0, \frac{\pi}{2}\right], \lambda, \mu \in[-2,1]
$$

is called a cubic trigonometric Bézier (T-Bézier) curve with two shape parameters $\lambda$ and $\mu$.

For any $\lambda, \mu \in[-2,1]$, from [13], we can see that the cubic T-Bézier basis (2) has the properties of partition of unity and nonnegativity, which implies that the corresponding cubic T-Bézier curve (14) has affine invariance and convex hull property. And for any $\lambda, \mu \in(-2,1]$, by Theorem 4 , we can see that the cubic T-Bézier basis (2) has the optimal totally positive property, which implies that the corresponding cubic T-Bézier curve (14) has the crucial property of variation diminishing; that is, no plane intersects a cubic T-Bézier curve more often than it intersects the corresponding control polygon.

Now we want to develop a new corner cutting algorithm for computing the cubic T-Bézier curve (14). For this purpose, for any $t \in[0, \pi / 2]$, let

$$
\begin{aligned}
& P_{0}^{1}=\frac{1-\lambda \sin t}{1+\sin t} P_{0}+\frac{(1+\lambda) \sin t}{1+\sin t} P_{1}, \\
& P_{1}^{1}=\frac{\cos t}{\sin t+\cos t} P_{1}+\frac{\sin t}{\sin t+\cos t} P_{2}, \\
& P_{2}^{1}=\frac{(1+\mu) \cos t}{1+\cos t} P_{2}+\frac{1-\mu \cos t}{1+\cos t} P_{3} .
\end{aligned}
$$

Then, we can rewrite the expression of the cubic T-Bézier curve (14) as follows:

$$
\begin{aligned}
T(t ; \lambda, \mu)= & \left(1-\sin ^{2} t\right)\left[(1-\sin t) P_{0}^{1}+\sin t P_{1}^{1}\right] \\
& +\left(1-\cos ^{2} t\right)\left[\cos t P_{1}^{1}+(1-\cos t) P_{2}^{1}\right]
\end{aligned}
$$


Furthermore, by setting

$$
\begin{aligned}
& P_{0}^{2}=(1-\sin t) P_{0}^{1}+\sin t P_{1}^{1}, \\
& P_{1}^{2}=\cos t P_{1}^{1}+(1-\cos t) P_{2}^{1},
\end{aligned}
$$

we have

$$
T(t ; \lambda, \mu)=\left(1-\sin ^{2} t\right) P_{0}^{2}+\left(1-\cos ^{2} t\right) P_{1}^{2} .
$$

The expressions (16) and (18) describe a corner cutting algorithm for computing the cubic T-Bézier curve (14). See Figure 1 for an illustration of this new developed algorithm.

\section{Conclusion}

The cubic trigonometric Bézier basis with two shape parameters $\lambda$ and $\mu$ given in [13] forms an optimal normalized totally positive basis of the space $T_{\lambda, \mu}$ for $\lambda, \mu \in(-2,1]$. However, the basis is not suited for curve design for $\lambda=-2$ or $\mu=-2$ from the blossom point of view. The new developed corner cutting algorithm is an efficient and stable process for computing the corresponding cubic trigonometric Bézier curves.

\section{Conflict of Interests}

The authors declare that there is no conflict of interests regarding the publication of this paper.

\section{Acknowledgments}

The authors would like to thank the anonymous reviewers for their valuable remarks for improvements. The research is supported by the National Natural Science Foundation of China under Grant nos. 60970097 and 11271376 and Graduate Students Scientific Research Innovation Project of Hunan Province, Grant no. CX2012B111.

\section{References}

[1] T. Lyche and R. Winther, "A stable recurrence relation for trigonometric B-splines," Journal of Approximation Theory, vol. 25, no. 3, pp. 266-279, 1979.

[2] G. Walz, "Identities for trigonometric $B$-splines with an application to curve design," BIT Numerical Mathematics, vol. 37, no. 1, pp. 189-201, 1997.

[3] G. Walz, "Trigonometric Bézier and Stancu polynomials over intervals and triangles," Computer Aided Geometric Design, vol. 14, no. 4, pp. 393-397, 1997.

[4] J. W. Zhang, "C-curves: an extension of cubic curves," Computer Aided Geometric Design, vol. 13, no. 3, pp. 199-217, 1996.

[5] J. W. Zhang, "Two different forms of $C$-B-splines," Computer Aided Geometric Design, vol. 14, no. 1, pp. 31-41, 1997.

[6] J. W. Zhang, F. L. Krause, and H. Y. Zhang, "Unifying Ccurves and $\mathrm{H}$-curves by extending the calculation to complex numbers," Computer Aided Geometric Design, vol. 22, no. 9, pp. 865-883, 2005.

[7] E. Mainar and J. M. Peña, "A basis of $C$-Bézier splines with optimal properties," Computer Aided Geometric Design, vol. 19, no. 4, pp. 291-295, 2002.
[8] X. L. Han, "Quadratic trigonometric polynomial curves with a shape parameter," Computer Aided Geometric Design, vol. 19, no. 7, pp. 503-512, 2002.

[9] X. L. Han, "Piecewise quadratic trigonometric polynomial curves," Mathematics of Computation, vol. 72, no. 243, pp. 13691377, 2003.

[10] X. L. Han, " $C^{2}$ quadratic trigonometric polynomial curves with local bias," Journal of Computational and Applied Mathematics, vol. 180, no. 1, pp. 161-172, 2005.

[11] X. Han, "Quadratic trigonometric polynomial curves concerning local control," Applied Numerical Mathematics, vol. 56, no. 1, pp. 105-115, 2006.

[12] X. L. Han, "Cubic trigonometric polynomial curves with a shape parameter," Computer Aided Geometric Design, vol. 21, no. 6, pp. 535-548, 2004.

[13] X. A. Han, Y. Ma, and X. Huang, "The cubic trigonometric Bézier curve with two shape parameters," Applied Mathematics Letters, vol. 22, no. 2, pp. 226-231, 2009.

[14] X. A. Han, X. L. Huang, and Y. C. Ma, "Shape analysis of cubic trigonometric Bézier curves with a shape parameter," Applied Mathematics and Computation, vol. 217, no. 6, pp. 2527-2533, 2010.

[15] R. J. Wu and G. H. Peng, "Shape analysis of planar trigonometric Bézier curves with two shape parameters," International Journal of Computer Science, vol. 10, no. 3, pp. 441-447, 2013.

[16] F. Ibraheem, M. Hussain, M. Z. Hussain, and A. A. Bhatti, "Positive data visualization using trigonometric function," Journal of Applied Mathematics, vol. 2012, Article ID 247120, 19 pages, 2012.

[17] U. Bashir and J. Md. Ali, "Data visualization using rational trigonometric spline," Journal of Applied Mathematics, vol. 2013, Article ID 531497, 10 pages, 2013.

[18] M. Hussain and S. Saleem, " $C^{1}$ rational quadratic trigonometric spline," Egyptian Informatics Journal, vol. 14, no. 3, pp. 211-220, 2013.

[19] M. Z. Hussain, M. Hussain, and A. Waseem, "Shape-preserving trigonometric functions," Computational and Applied Mathematics, 2013.

[20] M. L. Mazure, "Quasi-Chebyshev splines with connection matrices: application to variable degree polynomial splines," Computer Aided Geometric Design, vol. 18, no. 3, pp. 287-298, 2001.

[21] M. L. Mazure, "On dimension elevation in quasi extended Chebyshev spaces," Numerische Mathematik, vol. 109, no. 3, pp. 459-475, 2008.

[22] M. L. Mazure, "Which spaces for design?" Numerische Mathematik, vol. 110, no. 3, pp. 357-392, 2008.

[23] M. L. Mazure, "On a general new class of quasi Chebyshevian splines," Numerical Algorithms, vol. 58, no. 3, pp. 399-438, 2011.

[24] M. L. Mazure, "Quasi extended Chebyshev spaces and weight functions," Numerische Mathematik, vol. 118, no. 1, pp. 79-108, 2011.

[25] J. M. Carnicer, E. Mainar, and J. M. Peña, "Critical length for design purposes and extended Chebyshev spaces," Constructive Approximation, vol. 20, no. 1, pp. 55-71, 2003.

[26] J. M. Carnicer and J. M. Peña, "Shape preserving representations and optimality of the Bernstein basis," Advances in Computational Mathematics, vol. 1, no. 2, pp. 173-196, 1993.

[27] E. Mainar and J. M. Peña, "Corner cutting algorithms associated with optimal shape preserving representations," Computer Aided Geometric Design, vol. 16, no. 9, pp. 883-906, 1999.

[28] L. L. Schumaker, Spline Functions: Basic Theory, Cambridge University Press, Cambridge, UK, 3rd edition, 2007. 


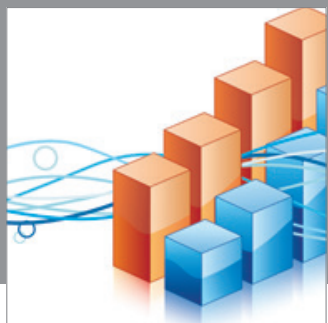

Advances in

Operations Research

mansans

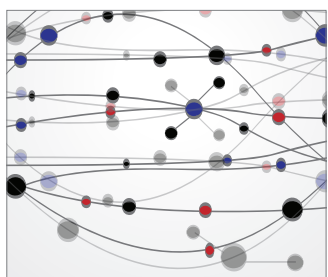

The Scientific World Journal
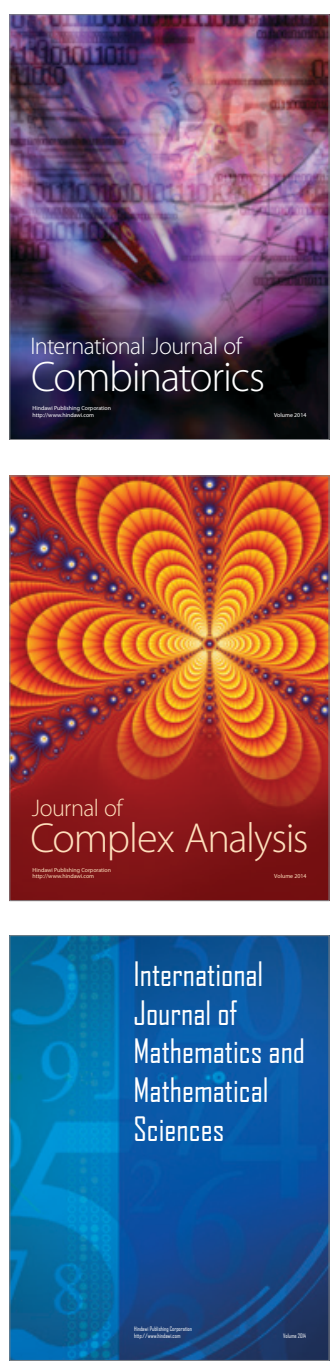
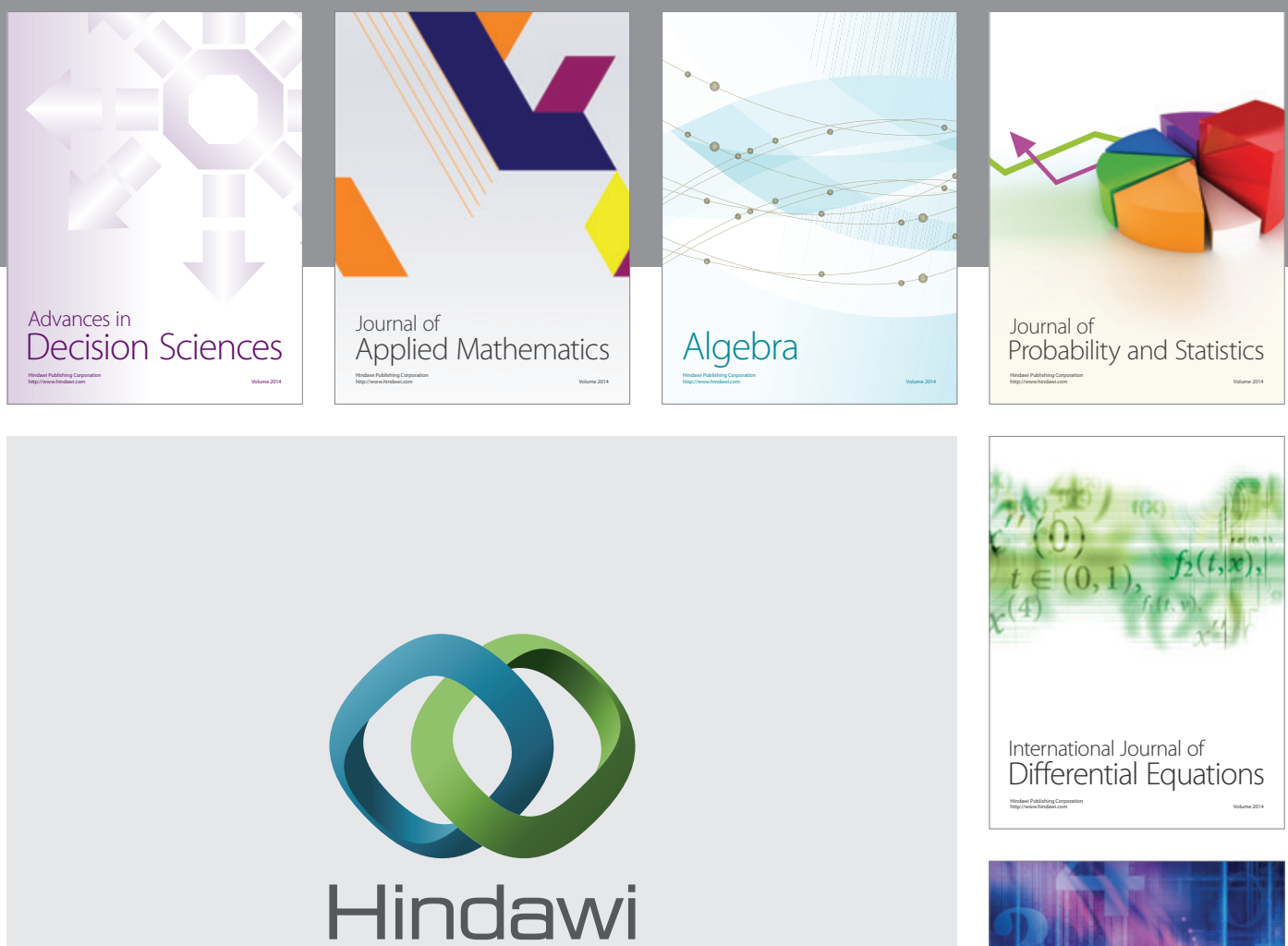

Submit your manuscripts at http://www.hindawi.com
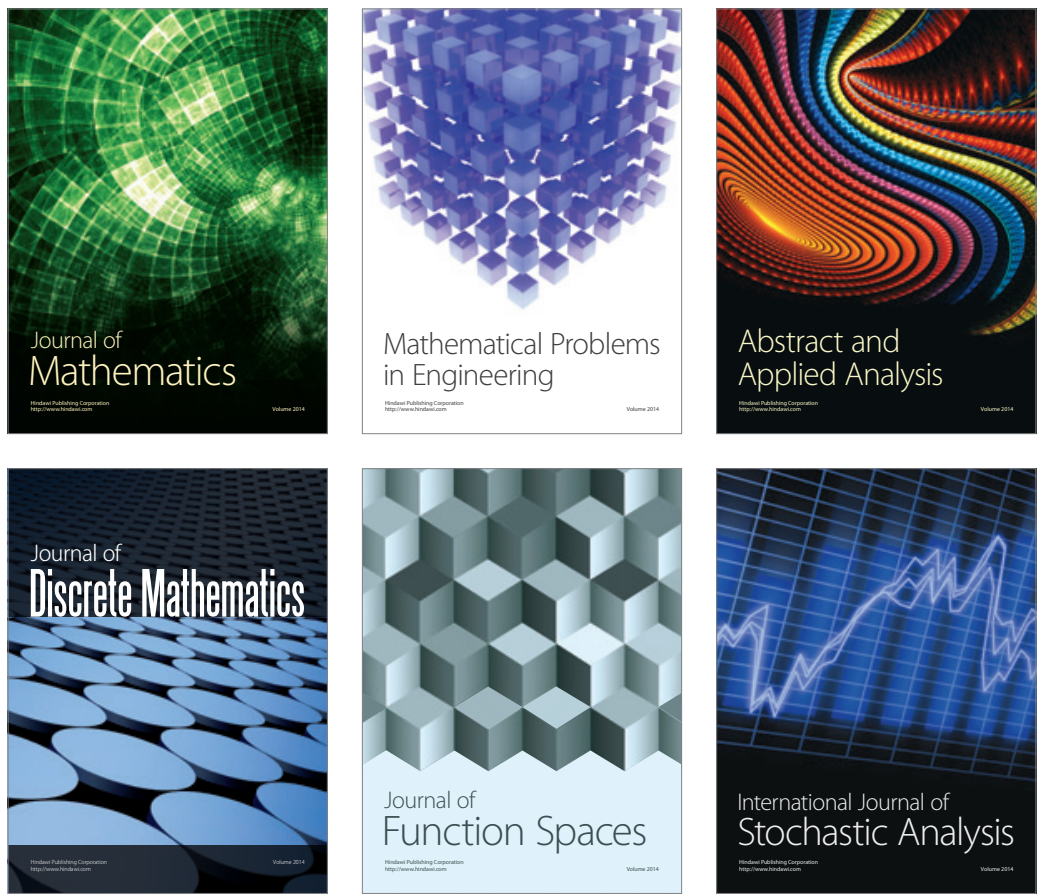

Journal of

Function Spaces

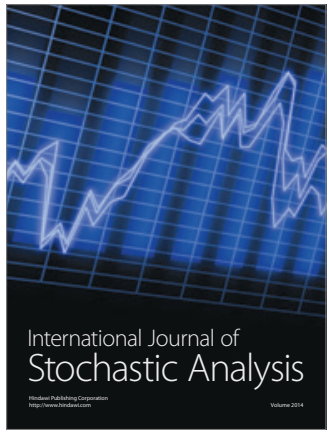

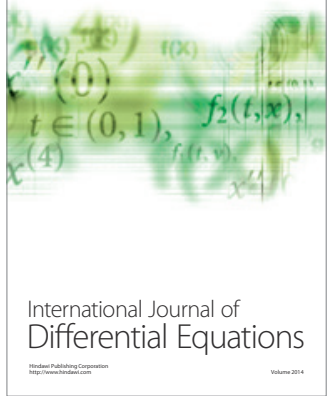
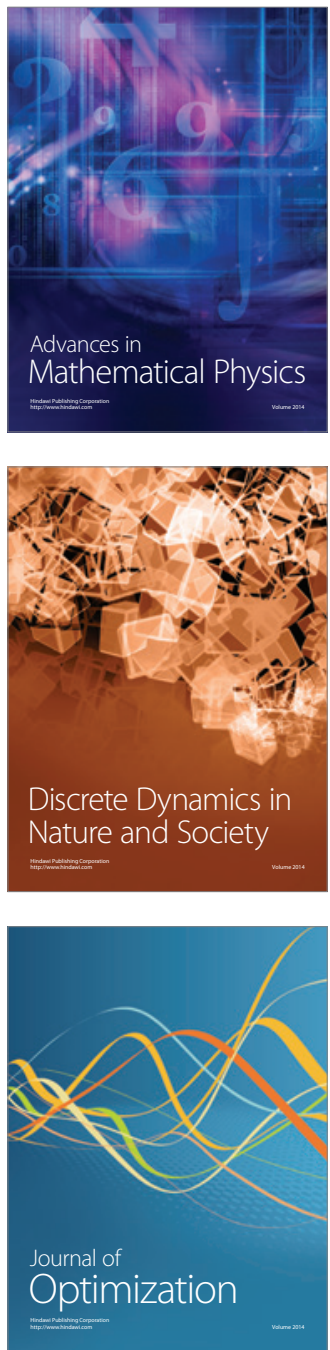